\title{
Use of Polyvinyl Alcohol as a Solubility Enhancing Polymer for Poorly Water-Soluble Drug Delivery (Part 2)
}

\author{
Chris Brough, ${ }^{1,2,4}$ Dave A. Miller, ${ }^{2}$ Daniel Ellenberger, ${ }^{1,2}$ Dieter Lubda, ${ }^{3}$ and Robert O. Williams III ${ }^{1}$
}

\begin{abstract}
Received 20 November 2015; accepted 28 January 2016; published online 10 February 2016
Abstract. The KinetiSol ${ }^{\circledR}$ Dispersing (KSD) technology has enabled the investigation into the use of polyvinyl alcohol (PVAL) as a concentration enhancing polymer for amorphous solid dispersions. Our previous study revealed that the $88 \%$ hydrolyzed grade of PVAL was optimal for itraconazole (ITZ) amorphous compositions with regard to solid-state properties, non-sink dissolution performance, and bioavailability enhancement. The current study investigates the influence of molecular weight for the $88 \%$ hydrolyzed grades of PVAL on the properties of KSD processed ITZ:PVAL amorphous dispersions. Specifically, molecular weights in the processable range of 4 to $18 \mathrm{mPa} \cdot \mathrm{s}$ were evaluated and the $4-88$ grade provided the highest AUC dissolution profile. Amorphous dispersions at 10, 20, 30, 40, and 50\% ITZ drug loads in PVAL 4-88 were also compared by dissolution performance. Analytical tools of diffusion-ordered spectroscopy and Fourier transform infrared spectroscopy were employed to understand the interaction between drug and polymer. Finally, results from a 30-month stability test of a $30 \%$ drug loaded ITZ:PVAL 4-88 composition shows that stable amorphous dispersions can be achieved. Thus, this newly enabled polymer carrier can be considered a viable option for pharmaceutical formulation development for solubility enhancement.
\end{abstract}

KEY WORDS: amorphous solid dispersion; itraconazole; polyvinyl alcohol; PVAL; solubility enhancement.

\section{INTRODUCTION}

One of the current challenges facing the pharmaceutical industry is addressing the increasing presence of poorly water-soluble drugs in commercial products and development pipelines (1-3). Using amorphous solid dispersions for oral delivery as a method to solve these challenges has increased in popularity in both commercial products and industry literature (4-7). For research purposes, itraconazole (ITZ) is often used as a model drug for concentration enhancement due to its aqueous solubility of approximately $1 \mathrm{ng} / \mathrm{mL}$ at neutral $\mathrm{pH}$ and approximately $4 \mu \mathrm{g} / \mathrm{mL}$ at $\mathrm{pH}$ $1.2(8-10)$. ITZ has been formulated in various polymeric carriers including hydroxypropylmethylcellulose (HPMC) (11,12), copovidone (PVPVA) (13), polyethyleneglycols (PEG) (14), polymethacrylates (15), hypromellose acetate succinate (HPMCAS) (16), hypromellose phthalate

\footnotetext{
${ }^{1}$ Division of Pharmaceutics, College of Pharmacy, The University of Texas at Austin, 1 University Station, Campus Mail Code A1902, Austin, Texas 78712, USA.

${ }^{2}$ DisperSol Technologies, LLC, 111 W. Cooperative Way, Building 3, Georgetown, Texas 78626, USA.

${ }^{3}$ Merck KGaA, Frankfurter Str. 250, D 64293, Darmstadt, Germany.

${ }^{4}$ To whom correspondence should be addressed. (e-mail: chris.brough@dispersoltech.com)
}

(HPMCP) (9), Soluplus® (17), polyvinyl acetate phthalate (PVAP), and cellulose acetate phthalate (CAP) (18).

The KinetiSol ${ }^{\circledR}$ Dispersing (KSD) technology is a thermal process that can create solid amorphous systems from challenging drugs and very highly viscous polymers (19). Utilizing this technology in our previous study, a broad range of molecular weights and degrees of hydrolysis of polyvinyl alcohol (PVAL) were investigated as a primary carrier in amorphous solid dispersion systems and for their solubility/ bioavailability enhancing effect on itraconazole. EMD Millipore's PVAL was utilized in both studies and the nomenclature for describing different polymer grades is $\mathrm{X}$-Y. X represents the viscosity (in $\mathrm{mPa} \cdot \mathrm{s}$ ) of the $4 \%$ aqueous solution at $20^{\circ} \mathrm{C}$, which is a relative indication of the molar mass and $\mathrm{Y}$ is the degree of hydrolysis of the polyvinyl acetate. An examination of the effects of molecular weight were conducted using PVAL 4-88, 26-88 and 40-88 with the 4-88 grade performing the best both from a KinetiSol ${ }^{\circledR}$ processing and dissolution enhancement point of view. Subsequently, 4-38, 4-75, 4-88 and 4-98 grades of PVAL were tested to ascertain the effect of the degree of hydrolysis for dissolution performance of ITZ. PVAL 4-38 did not fully release due to the fact that polyvinyl acetate, the predominant component of the grade, is less water-soluble than PVAL. PVAL 4-98 also did not fully release due to the highly crystalline nature of the fully hydrolyzed grade which swelled in the aqueous environment, but 
did not completely dissolve. Both PVAL 4-75 and 4-88 released fully in the acidic portion of the dissolution test, but after the $\mathrm{pH}$ change to the neutral media the 4-88 grade provided best maintenance of supersaturated drug concentrations.

Research conducted on various molecular weights of HPMC on the dissolution performance of ITZ show that higher molecular weights are more efficient stabilizers of drug supersaturation after the $\mathrm{pH}$ change from the acidic gastric condition to the neutral intestinal environment $(9,20)$. Similar to HPMC, PVAL has a wide spectrum of molecular mass from low to high viscosity ranges and it is anticipated that it will have an effect on the performance of the amorphous dispersion. Previous experimentation of PVAL 26-88 and 40-88 showed that these grades are excessively viscous as to pose processing challenges, which resulted in uneven drug distribution within the polymer. However, there are several grades between $4-88$ and 26-88, namely 5-88, 8-88, $18-88$ and 23-88, which could be investigated to determine if molecular weight effects post $\mathrm{pH}$ change dissolution performance. The current research comparatively evaluated the PVAL grades within a processable viscosity range (4-88 to $18-88$ ) for their effect on the properties of amorphous dispersions of ITZ prepared by KSD.

Rendering a drug amorphous within a semi-crystalline polymeric carrier does raise concerns for the long term storage stability of the formulation (21-25). Since the drug thermodynamically prefers the crystalline state, the polymeric carrier must prevent this occurrence through steric hindrance and/or molecular interaction. The concern of semi-crystalline polymers is that structured molecular geometry allows polymer rich and drug rich domains because drug is solubilized in the amorphous polymer regions. Increased drug concentration lowers the steric hindrance provided by the polymer. Additionally, polymer crystal growth can occur over time and hence the drug must compete with the polymer itself for molecular interaction stability. PVAL does have at least one advantage over other semi-crystalline polymers like polyethylene glycol (PEG) in that it does have a higher $T_{g}$ and $T_{m}$, which indicates lower molecular mobility at temperature ranges associated with physical stability assuming drug/polymer miscibility in the dispersion.

PVAL is known for providing solution stabilization as it is used in the pharmaceutical industry both as a stabilizing agent for emulsions (26) and as an emulsifier in creating PLGA nanoparticles $(27,28)$. The previous research illustrated in both in vivo and in vitro studies PVAL's ability to increase free drug concentrations, and consequently oral absorption of the small molecule, itraconazole. Because of this performance, it is hypothesized that solution interaction exists between ITZ and PVAL and can be characterized. In an attempt to determine the mechanism of interaction that allows this apparent solubility increase, the current study employed the analytical tools of Fourier transform infrared spectroscopy (FTIR) and diffusion-ordered spectroscopy (DOSY).

\section{MATERIALS}

Itraconazole was purchased from Neuland Laboratories Limited (India). All grades of PVAL (EMD Millipore) were donated by Merck Millipore. All other chemicals used in this study were ACS grade.

\section{METHODS}

\section{KinetiSol ${ }^{\circledR}$ Dispersing (KSD)}

Compositions for this study were produced by a lab scale, GMP pharmaceutical compounder designed and manufactured by DisperSol Technologies, L.L.C. (Georgetown, TX, USA). Prior to KSD processing, materials were weighed, dispensed into a polyethylene bag, manually shaken for approximately $1 \mathrm{~min}$, and charged into the compounder chamber. During processing, computer controls monitor processing parameters in real-time and eject the material at a preset ejection temperature. Discharged material was immediately quenched in a cooling die under pressure in a pneumatic press. Cooled material was then cryomilled in a SPEX 6870 Freezer/Mill, with a 2 cycle run of 5 min each at 10 cycles per second after a 3 min cool time and 2 min timer between cycles. All further analyses were conducted on this powder.

\section{Powder X-Ray Diffraction (XRD)}

An Inel Equinox 100 X-ray diffractometer (INEL, Artenay, France) was used to detect the presence of ITZ crystallinity. Milled compositions, physical mixtures or unprocessed ITZ were loaded on a rotating aluminum sample holder and placed in the radiation chamber. The Equinox 100 utilizes $\mathrm{Cu} \mathrm{K}$ Alpha radiation $(\lambda=1.5418 \AA$ ) with a curved radius detector to simultaneously measure a $2 \Theta$ range of 5$110^{\circ}$. Operating voltage and amperage were adjusted to $41 \mathrm{kV}$ and $0.8 \mathrm{~mA}$, respectively and the scan time for each sample was $10 \mathrm{~min}$.

\section{Polarized Light Microscopy (PLM)}

PLM analysis was conducted on a Meiji Techno MT 9300 polarizing light microscope with a first order red compensator. Pulverized samples were dusted on a glass slide and viewed at $400 \times$ magnification. The slide holder was rotated at least 90 degrees while being observed to detect any light refractions.

\section{High-Performance Liquid Chromatography (HPLC)}

The ITZ content in processed samples and dissolution aliquots were analyzed with a Dionex Ultimate 3000 H/UPLC system equipped with diode array detector extracting at $263 \mathrm{~nm}$. The system was operated under isocratic conditions with a 70:30:0.05 acetonitrile:water:diethanolamine mobile phase at a flow rate of $1.0 \mathrm{~mL} / \mathrm{min}$. The column used for analysis was a Phenomenex Luna $5 \mu \mathrm{m} \mathrm{C18(2)} 100 \AA$, $150 \mathrm{~mm} \times 4.6 \mathrm{~mm}$ (Phenomenex, Torrance, CA, USA) HPLC column. Dionex Chromeleon 7.2 software was used to analyze all chromatography data.

\section{Non-sink Dissolution Analysis}

Non-sink dissolution analysis was conducted with a VK 7000 dissolution tester (Varian, Inc., Palo Alto, CA, USA) configured as Apparatus 2. The test was performed similar to USP XXXVII (38) dissolution for delayed release dosage forms. Solid dispersions $(n=3)$ were weighed to achieve a 
mass equivalent to $37.5 \mathrm{mg}$ ITZ and dispensed in dissolution vessels on the surface of $750 \mathrm{~mL}$ of $0.1 \mathrm{~N} \mathrm{HCl}(\sim 10 \times$ ITZ equilibrium solubility in acid) equilibrated to a temperature of $37.0 \pm 1^{\circ} \mathrm{C}$ with a paddle rotation of $50 \mathrm{rpm}$. After $2 \mathrm{~h}$, a buffer medium of $250 \mathrm{~mL}$ of $0.2 \mathrm{M} \mathrm{Na}_{3} \mathrm{PO}_{4}$, preheated to $37.0 \pm 1^{\circ} \mathrm{C}$, was added to the dissolution vessels to adjust the $\mathrm{pH}$ to 6.80 .

Drug content in solution was directly measured with a fiber optic Spectra ${ }^{\mathrm{TM}}$ instrument (Pion Inc., Billerica, MA, USA) fitted with $5 \mathrm{~mm}$ path length Pion Probes. Readings were recorded every $5 \mathrm{~min}$ over the entire duration. Additionally, $5 \mathrm{~mL}$ samples were taken at 2:00, 2:05, 2:10, 2:15, 2:30, 3:00, 3:30, 4:00 and 5:00 hours. Samples were immediately filtered using $0.2 \mu \mathrm{m}$ PVDF with GMF membrane, $13 \mathrm{~mm}$ filters, diluted at a 1:1 ratio with mobile phase, mixed and then transferred into $1.5 \mathrm{~mL}$ vials for HPLC analysis. Pion data was correlated to match HPLC results by adjusting the 2nd derivative calculation range parameters to a span between 230 and $250 \mathrm{~nm}$ until values became equivalent.

\section{Precipitation Inhibition Analysis}

Solid dispersions $(n=3)$ were weighed to achieve an equivalent of $37.5 \mathrm{mg}$ ITZ and placed in suitable vessels containing $750 \mathrm{~mL}$ of $0.1 \mathrm{~N} \mathrm{HCl}$ (to match concentrations in non-sink dissolution studies) that had been equilibrated to a temperature of $37.0 \pm 1^{\circ} \mathrm{C}$. Containers were placed on stirring tables and agitated vigorously for several hours until solutions became clear. Media was then transferred to dissolution vessels within a VK 7000 dissolution tester (Varian, Inc., Palo Alto, CA, USA) configured as Apparatus 2 with a paddle rotation of $50 \mathrm{rpm}$. A buffer medium of $250 \mathrm{~mL}$ of $0.2 \mathrm{M}$ $\mathrm{Na}_{3} \mathrm{PO}_{4}$, preheated to $37.0 \pm 1^{\circ} \mathrm{C}$, was added to the dissolution vessels to adjust the $\mathrm{pH}$ to 6.80 . Samples were taken immediately before $\mathrm{pH}$ change, then after the $\mathrm{pH}$ change at 5, 10, 15, 30, 60, 90, 120, and $180 \mathrm{~min}$. Samples were immediately filtered using $0.2 \mu \mathrm{m}$ PVDF with GMF membrane, $13 \mathrm{~mm}$ filters, diluted to a $1: 1$ ratio with mobile phase, mixed and then transferred into $1.5 \mathrm{~mL}$ vials for HPLC analysis.

\section{Differential Scanning Calorimetry (DSC)}

Modulated DSC analysis was conducted using a TA Instruments Model mQ20 DSC (New Castle, DE) equipped with a refrigerated cooling system and autosampler. Samples were weighed to $8-10 \mathrm{mg}$ in aluminum-crimped pans. Samples $(n=3)$ were heated at a ramp rate of $5^{\circ} \mathrm{C} / \mathrm{min}$ from 0 to $215^{\circ} \mathrm{C}$ with a modulation temperature amplitude of $1.0^{\circ} \mathrm{C}$ and a modulation period of $60 \mathrm{~s}$ for all studies. Ultrahigh purity nitrogen was used as the purge gas at a flow rate of $50 \mathrm{~mL} / \mathrm{min}$. All data analyses were performed using TA Universal Analysis software.

\section{Fourier Transform Infrared Spectroscopy (FTIR)}

Samples were tested in a Thermo Scientific Nicolet iS50 FTIR Spectrometer (Thermo Electron Scientific Instruments LLC, Madison, WI, USA) with the Omni-Sampler module for attenuated total reflectance (ATR). The scan range was 2000700 wavenumber $\left(\mathrm{cm}^{-1}\right)$ at a resolution of $4 \mathrm{~cm}^{-1}$ for 32 scans per sample. Data collection and analysis were performed with OMNICTM.

\section{Diffusion-Ordered Spectroscopy (DOSY)}

Amorphous dispersions and PVAL were dissolved in deuterated solutions of $0.1 \mathrm{~N} \mathrm{DCl}$ prepared by diluting concentrated DCL with $\mathrm{D}_{2} \mathrm{O}$. Samples were tested in a Varian VNMRS $600 \mathrm{MHz}$ nuclear magnetic resonance (NMR) spectrometer (Palo Alto, CA, USA) for solution with a Doneshot pulse sequence. The spectrum was adjusted until water at $25^{\circ} \mathrm{C}$ was $19.02 \times 10^{-10}$ and all data generated is correlated to that reference. Figures were generated using Varian VNMRJ software.

\section{RESULTS AND DISCUSSION}

\section{Evaluation of PVAL Molecular Weight}

To be consistent with our previous study, a drug loading of $20 \%$ ITZ was selected for the preparation of amorphous dispersions with PVAL grades of 4-88, 5-88, 8-88, and 18-88. All acceptable KSD compositions were verified to be amorphous by XRD and PLM, and HPLC analysis was conducted to establish dispersion potencies (data not shown). For dissolution testing, the samples were weight adjusted according to potency to achieve an equivalent amount of ITZ in each vessel. Figure 1 contains the dissolution curves of the listed PVAL grades for the 2-h pH change dissolution method. Error bars are not shown due to data density making them indistinguishable.

Both PVAL 4-88 and 5-88 grades fully released ITZ at supersaturated levels with little variance between the two in the acid phase. The pulverized formulations were introduced on the top of the dissolution media; then powder broke through the surface tension and dissolved as the particles sank. PVAL 8-88 and 18-88 were not able to achieve complete release, which was observed during the test with powder sinking to the bottom of the dissolution vessels without dissolving after approximately $1 \mathrm{~h}$. After the $\mathrm{pH}$ change the precipitation inhibition performance followed the reverse order of molecular weights: 4-88 $>5-88>8-88>18-88$.

Prior to the $\mathrm{pH}$ change, $8-88$ had only reached $70 \%$ of drug in solution and $18-88$ had only attained $60 \%$. It was speculated that if these two grades could achieve $100 \%$ drug release the rank order of performance might change. An alternative dissolution study using the same amorphous dispersions, called precipitation inhibition analysis, was conducted which forced complete drug release from all polymer grades prior to the $\mathrm{pH}$ change with the results exhibited in Fig. 2.

Even with the formulations at the same starting point, the lower molecular weights outperformed the higher ones in reducing the rate of drug precipitation. These results match the previous study where PVAL 4-88 provided better performance than combinations containing the 26-88 and 40-88 grades. Thus, contrary to the trends observed with HPMC (20), for PVAL the lower molecular weights have superior dissolution performance over higher molecular weights.

This trend is confirmed by comparing the rates of dissolution for the polymers themselves in water. Product literature shows that lower molecular weights of the -88 grades have faster dissolution rates specifically in the temperature range between 20 and $40^{\circ} \mathrm{C}(29)$. Additionally, as shown in that same 


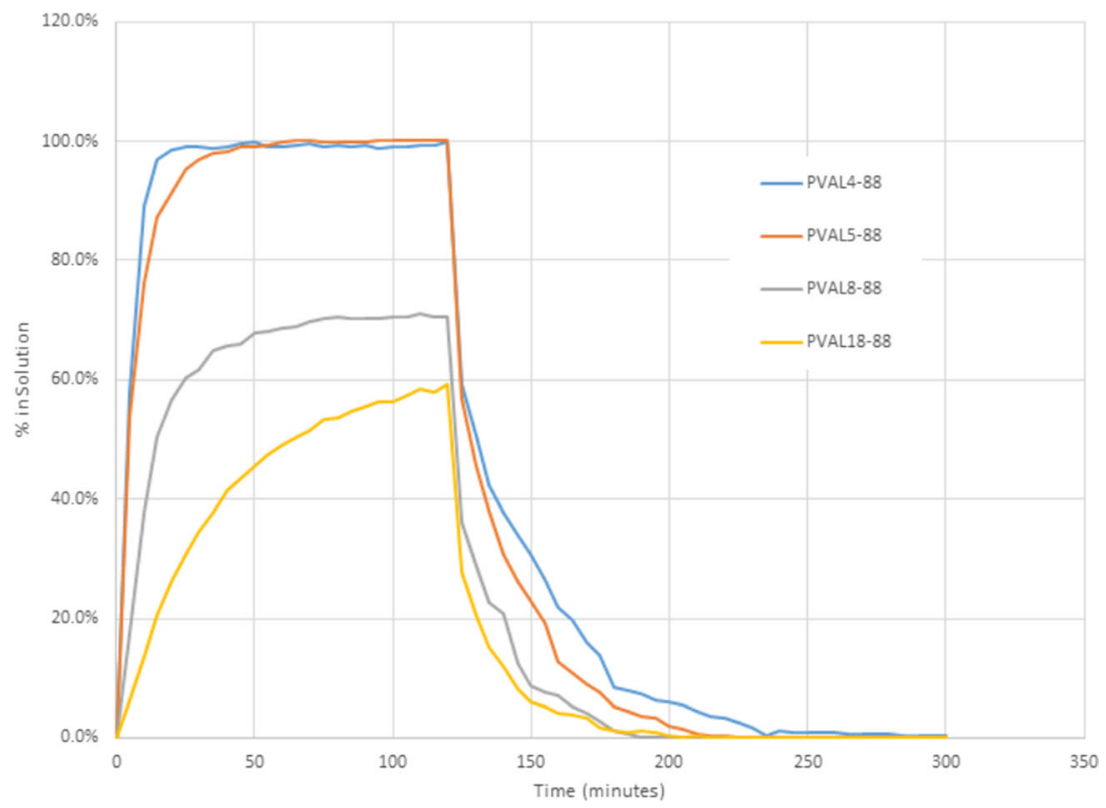

Fig. 1. Dissolution profiles of $20 \%$ ITZ in PVAL 4-88, 5-88, 8-88, and 18-88

product literature, PVAL reduces interfacial tension, particularly the surface tension of water with respect to air, with differing effects according to molecular weight as seen in Figure 24 of the citation. That figure specifically compares PVAL grades $40-88,18-88,8-88$, and $4-88$ for reduction in water surface tension. The rank order of greatest surface tension reduction to lowest is $4-88>8-88>18-88>40-88$. Thus, the lower molecular weights have a greater reduction in surface tension of water.

Since amorphous dispersion powders were dispensed on the surface of the media in the vessels for the non-sink dissolution test, the lower surface tension and faster dissolution rate of the lower molecular weight grades would speak to the faster dissolution rate of ITZ. The effect on water surface tension indicates that lower molecular weight grades effectively functions as a polymeric surfactant more than higher molecular weights. Polymeric surfactants have been shown to create micelle or other geometries in solution to stabilize drug concentrations (30) and also have been demonstrated to stabilize water-insoluble drugs in solution $(31,32)$. This would support the results for the precipitation inhibition analysis where the lower molecular weights were more effective in reducing the rate of drug precipitation.

\section{Drug Loading Evaluation}

All dissolution studies from the current and previous research have been conducted with $20 \%$ drug loading. It is noted that the drug loading of ITZ:HPMC in the commercial product Onmel ${ }^{\mathrm{TM}}$ and the coating for the nonpareil beads in Sporanox ${ }^{\circledR}$ which is also ITZ in HPMC are both $40 \%$ (33). Typically, the higher the drug load, the lower the dissolution performance because the ratio of drug to polymer decreases and solubility enhancement trends with increasing polymer

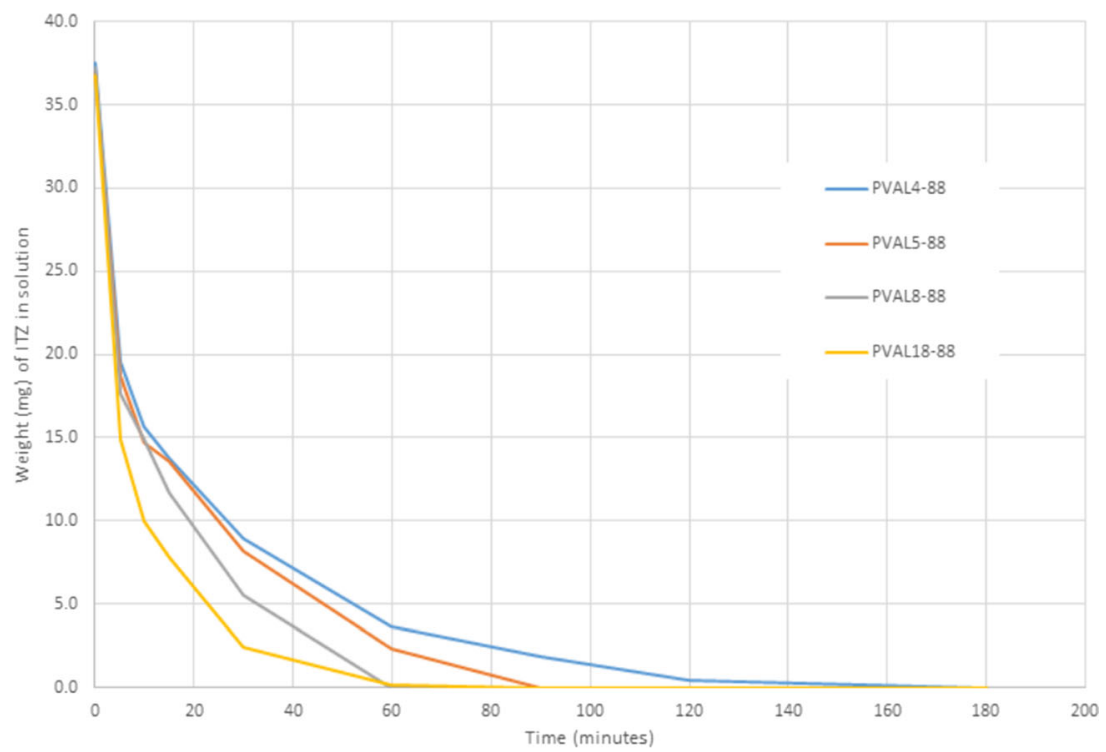

Fig. 2. Precipitation inhibition curves of $20 \%$ ITZ in PVAL 4-88, 5-88, 8-88, and 18-88 
concentration (34). However, if higher drug loading can be achieved, the size of the dosage form can be reduced, typically improving patient convenience and compliance. Hence, optimal drug loading is a balance between performance and size of the final dosage form.

To investigate the effect of drug loading on dissolution performance, amorphous dispersions of ITZ in PVAL 4-88 were produced at 10, 20, 30, 40, and 50\% drug loads. HPLC analysis measured actual potencies and samples were weight adjusted to ensure $37.5 \mathrm{mg}$ of drug were contained in each dissolution vessel. The result from the non-sink dissolution test is found in Fig. 3.

The rank order from highest dissolution performance in acid is as follows: $20 \%>30 \%>40 \%>10 \%>50 \%$. Surprisingly, the $10 \%$ dispersion did not have the highest rate of dissolution. It exhibited a faster initial rate than 30 and $40 \%$, but slowed after the initial $20 \mathrm{~min}$ to perform only better than the $50 \%$ loading. Only the 10 and 50\% drug loading did not achieve $100 \%$ drug release within the 2 -h window of the acid portion of the experiment.

At the end of the 2-h acid phase, there was still powder floating at the top of the vessel of the $10 \%$ drug load sample. Keeping the relative amounts in perspective can provide a probable explanation for this behavior. The $10 \%$ drug loading has a theoretical weight of $375 \mathrm{mg}$; the $20 \%$ drug loading has $187.5 \mathrm{mg}$ weight on down to the $50 \%$ drug loading only having $75 \mathrm{mg}$ of total amorphous intermediate powder. The $187.5 \mathrm{mg}$ of $20 \%$ ITZ loaded powder floated on top (like all other samples) initially, then within a few minutes dispersed into the vessel and was dissolved within $20 \mathrm{~min}$. Both the 30 and $40 \%$ loaded samples followed the same pattern, but with longer timeframes. Since PVAL functions as a viscosity increasing agent and has been used in forming hydrogels (35), the large amount of the $10 \%$ ITZ dispersion could create a viscous solution on the top of the dissolution media to prevent all of the powder from dissolving. This viscous solution could have also have prevent drug from dispersing uniformly within the vessel in the 2-h timeframe. Since both the Pion and HPLC samples were taken approximately $1 / 3$ of the distance from the bottom of the vessel, this would have resulted in lower drug concentration readings. Pouring the buffer into the dissolution vessel would have aided in stirring and dispersing the drug throughout the vessel, which explains why the $10 \%$ drug loading went from the 2nd lowest concentration to the 2 nd highest after the $\mathrm{pH}$ change. Since a $10 \%$ drug is not desired as a commercial loading, the method was not altered to achieve a more accurate dissolution profile.

For the $50 \%$ drug loading sample, the remaining powder from the acid phase was on the bottom of the dissolution. Like the $20-40 \%$ loaded samples, the powder that was introduced on the top surface of the vessel dispersed into the dissolution media. However, instead of all the particles dissolving on the decent, some of the particles sank to the bottom of the vessel. The structural transitions of PVAL during dissolution have been studied and it was discovered that below $45^{\circ} \mathrm{C}$, water molecules mainly diffuse into the amorphous region of the polymer (36). Since the amorphous region in polymers is also responsible for the solubilization of small molecules (37), the $50 \%$ drug loading appears to be sufficiently high in some particles to prevent water diffusion and, thus, block dissolution.

After the $\mathrm{pH}$ change the rank order follows as was expected with $10 \%>20 \%>30 \%>40 \%>50 \%$. Ten percent ITZ did start at a lower concentration than the $20 \%$, but had higher actual amounts of drug in solution at $180 \mathrm{~min}$ which was an hour after the $\mathrm{pH}$ change. The $20 \%$ ITZ composition had the fastest dissolution rate and reduced the rate of precipitation at a similar level to the $10 \%$ composition. The $30 \%$ loading was a close second to $20 \%$ in the acid phase dissolution and exhibited a rate of precipitation only slightly lower than both the 10 and $20 \%$ compositions. Since, optimal drug loading is a balance between performance and size of the final dosage form, the $30 \%$ loading is probably closer to the optimal drug loading than all the other drug loadings tested.

Thermal analysis in the form of DSC was performed on the five different drug loads as seen in Fig. 4. A reversing heat flow thermogram was produced (data not shown) to verify that all compositions have a composite glass transition temperature at the same approximate temperature. This is presented in Fig. 4 with a representative value on the $20 \%$ ITZ

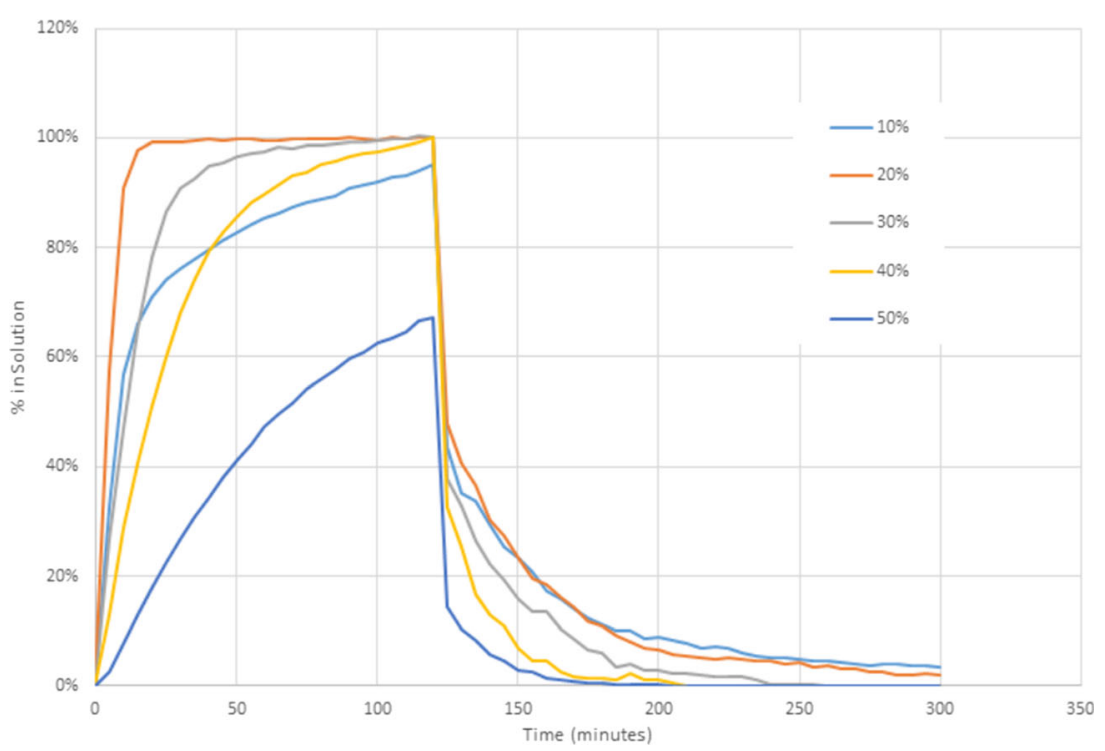

Fig. 3. Dissolution comparison of different ITZ loadings in PVAL 4-88 


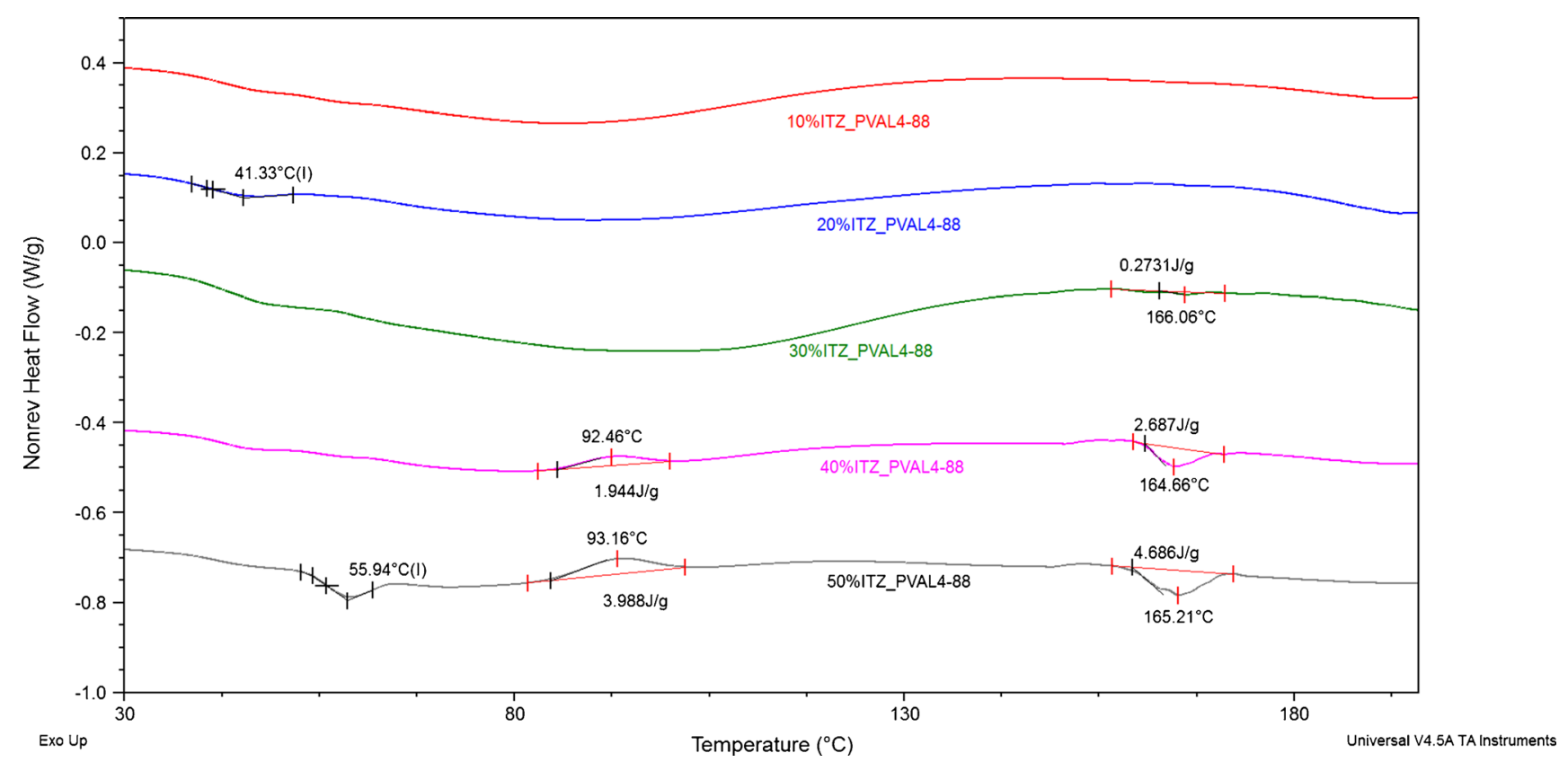

Fig. 4. Non-reversing heat flow DSC thermograms for $10-50 \%$ ITZ loaded amorphous dispersions in PVAL 4-88

line at $41.33^{\circ} \mathrm{C}$; all other compositions have the event at a similar temperature but were not labeled on the graph to prevent encumbrance. The $30 \%$ ITZ thermal profile exhibits an endothermic even at $166.06^{\circ} \mathrm{C}$, which indicates the presence of crystalline ITZ. It was shown in the previous study that DSC testing causes some fraction of the ITZ to recrystallize. This phenomenon is more clearly visible in the 40 and $50 \%$ compositions where the recrystallization exothermic events are evident at 92.46 and $93.16^{\circ} \mathrm{C}$, respectively. They also have endotherms showing crystalline ITZ at 164.66 and $165.21^{\circ} \mathrm{C}$, respectively. The magnitude of these events for the $50 \%$ composition is approximately double that of the $40 \%$ composition displaying the $50 \%$ composition's decreased capacity for stabilizing amorphous ITZ. The $50 \%$ also has a unique thermal event at $55.94^{\circ} \mathrm{C}$, which was determined in the previous study to be the $\mathrm{T}_{\mathrm{g}}$ of amorphous itraconazole. It appears the 50\% loading has exceeded PVAL's amorphous regions' capability to solubilize drug and drug rich domains of amorphous ITZ are present. This result supports dissolution performance where drug saturation in PVAL's amorphous regions prohibited water diffusion and, thus, the composition was not able to achieve $100 \%$ drug release. Additionally, the DSC results also confirm the selection of the $30 \%$ ITZ composition as the closest to optimal because it did not experience a substantial recrystallization event like the 40 and $50 \%$ formulations.

\section{Evaluation of the ITZ/PVAL Interaction}

Because the ITZ is amorphous in the solid dispersion, it is not surprising to see apparent solubility levels much higher than the crystalline equilibrium solubility. However, without the aid of a solubility enhancing/stabilizing polymer, the thermodynamically unstable supersaturation would soon return to the crystalline equilibrium solubility. Especially, as the aqueous environment transitions from the acid gastric environment to the $\mathrm{pH}$ neutral environment of the intestinal tract, a weakly basic drug like ITZ would return to solubility equilibrium quite rapidly (20). Dissolution profiles contained in this and the previous study report concentration levels as high as 8000 times the saturation concentration in neutral media and maintaining at least 4000 times the saturation concentration for $30 \mathrm{~min}$ after the $\mathrm{pH}$ change. These results suggest an attractive interaction between the ITZ and PVAL.

FTIR-ATR has been reported as an analytical tool to investigate such possible interactions (38-40). Peaks in the spectra can be associated with specific atomic bonding and shifting or changing shape can indicate molecular interactions. Figure 5 contains the FTIR spectra of amorphous itraconazole, preprocessed PVAL 4-88 and amorphous solid dispersions of 10 and 20\% ITZ loading in PVAL 4-88.

The result for ITZ is similar to published literature $(41,42)$ and the PVAL result is consistent with product literature $(29,35)$. For PVAL, the broad peak at $3330 \mathrm{~cm}^{-1}$ is associated with $\mathrm{O}-\mathrm{H}$ stretching, the small peak at $2941 \mathrm{~cm}^{-1}$ with $\mathrm{C}-\mathrm{H}$ stretching, the $1732 \mathrm{~cm}^{-1}$ peak with $\mathrm{C}=\mathrm{O}$ groups (from unhydrolyzed ester groups), the peak at $1429 \mathrm{~cm}^{-1}$ with $\mathrm{CH}_{2}$ bending, the $1251 \mathrm{~cm}^{-1}$ peak with the $\mathrm{C}-\mathrm{O}-\mathrm{C}$ groups (also from unhydrolyzed ester groups) and, finally, the $1093 \mathrm{~cm}^{-1}$ peak is associated with $\mathrm{C}-\mathrm{O}$ stretch and $\mathrm{O}-\mathrm{H}$ bending. For ITZ, the first major peak at $1700 \mathrm{~cm}^{-1}$ is associated with $\mathrm{C}=\mathrm{O}$ stretching, the $1511 \mathrm{~cm}^{-1}$ peak is related with $\mathrm{CO}-\mathrm{NH}_{2}$, and the $946 \mathrm{~cm}^{-1}$ linked with $\mathrm{C}-\mathrm{Cl}$. Most peaks do not show any difference between the individual components and the amorphous dispersions. Yet, the broad peak associated with $\mathrm{O}-\mathrm{H}$ stretching on PVAL showed a shift from $3330 \mathrm{~cm}^{-1}$ to 3334 and $3335 \mathrm{~cm}^{-1}$ on the 10 and $20 \%$ ITZ loading in PVAL 4-88, respectively. The ITZ peak at $1700 \mathrm{~cm}^{-1}$ that correlates to $\mathrm{C}=\mathrm{O}$ stretching, shows a change of 1713 and $1706 \mathrm{~cm}^{-1}$ for the same respective 10 and $20 \%$ ITZ loaded amorphous dispersions. While it does appear that hydrogen bonding might be occurring, the magnitude of the shifts advocates that interactions, more than just hydrogen bonding alone, would account for the storage stability of the 


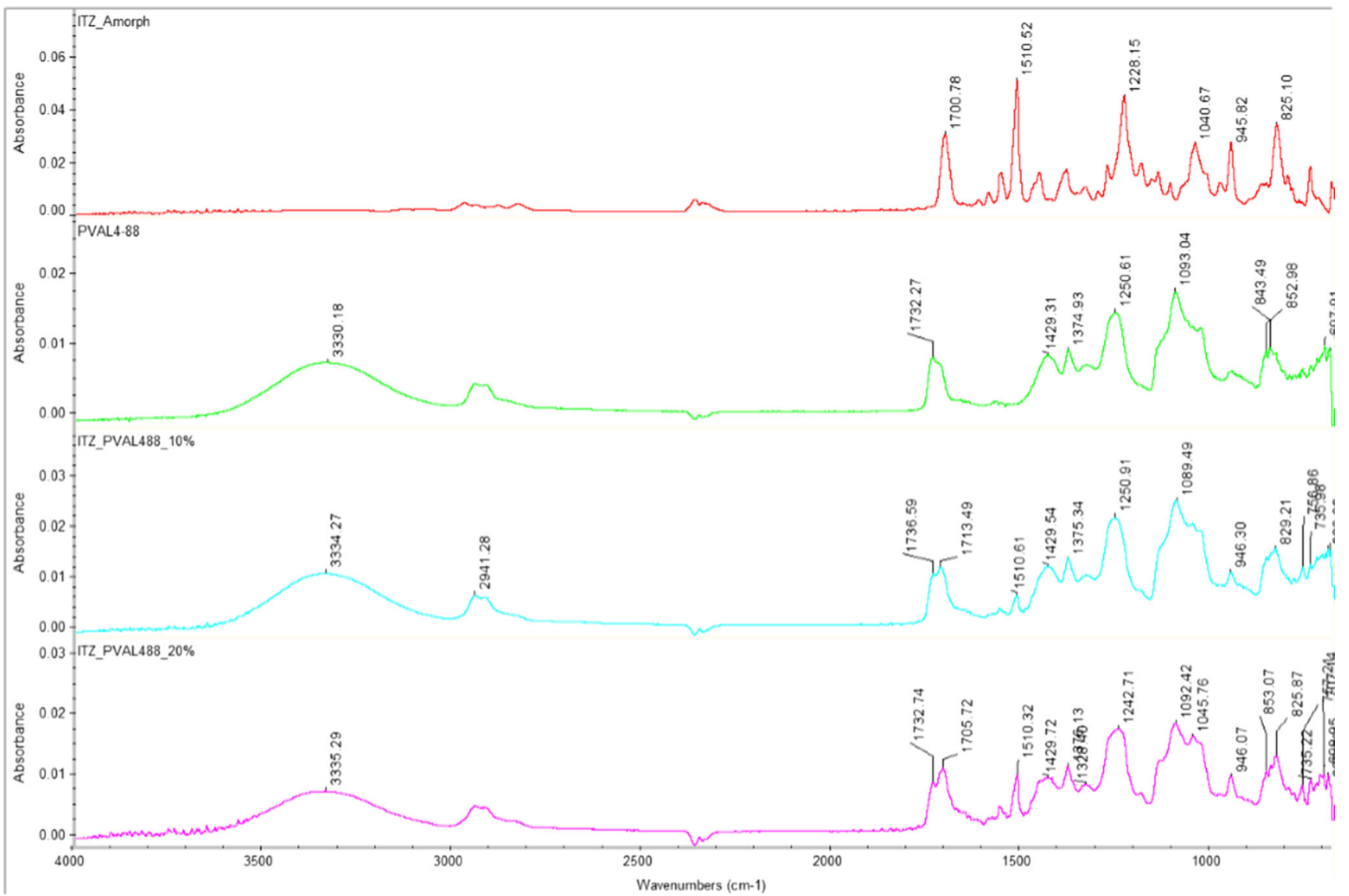

Fig. 5. FTIR-ATR spectra of amorphous ITZ, PVAL 4-88, and amorphous dispersions

amorphous dispersion. Since FTIR is a solid-state tool, it is not known if these same interactions would occur in solution but might indicate initial interaction for early dissolution.

Diffusion-ordered spectroscopy (DOSY) or sometimes called diffusion-ordered NMR spectroscopy and diffusionordered correlation spectroscopy has been used to determine the drug complexation with cyclodextrins (43), drug release from a hydrogel matrix (44), identification of fake formulations of commercial products (45), and to see interactions of polymers and drugs in solution (46). DOSY is a measure of diffusion and can distinguish different components in a formulation by the rate of diffusion and location on the spectra. Small molecules have a faster diffusion rate than large polymers and will have a different measurement of diffusion unless they are strongly bound by a covalent, ionic or hydrogen bond, which would give them the same measurement of diffusion as they act as single entity. Figure 6 has the DOSY map for PVAL 4-88 only and for the $20 \%$ drug loading in PVAL488.

The Y or F1 axis is the measurement of diffusion or how well the molecules travel within the solution with lower numbers at the top representing slower diffusion and increasing with the numbers to reach the highest diffusion rates at the bottom. The X or F2 axis helps track any chemical shift from the top spectra. Figure $6 a$ is a map of pure PVAL 4-88 that has not been processed. It is interesting to note that the polymer has two different diffusion coefficients, with the first band around 0.4 and the second one around 1.0 on the F1 axis. Product literature speaks specifically about PVAL's propensity toward complex formation in the presence of certain acids or salts (29). Since the solution is $0.1 \mathrm{~N} \mathrm{DCl}$, it is possible to have PVAL form a bimolecular complex. Additionally, in the discussion regarding PVAL reduction of water surface tension, it was mentioned that PVAL 4-88's greater effect on surface tension would allow it to function with surfactant-like properties, which would allow for molecules to self-associate in solution. Thus, it appears that the two different diffusion coefficients are formed by some molecules going into solution in groups and other by themselves. Whatever the actual cause is for the grouping phenomena, it appears that acetate groups on the PVAL molecular chain play a part because the spectra associated with acetate (around 2 on the F2 axis) is only observed in the slower diffusion group. Also on Fig. $6 a$ is an area that is around 10 on the F1 axis and around 2 on the F2 axis; this charts the residual acetyl groups from the hydrolysis process that created the polymer.

Figure $6 \mathrm{~b}$ is a map of the amorphous dispersion containing 20\% ITZ in PVAL4-88. At the top, again, are the two different diffusion coefficients of the PVAL 4-88. Around 3 on the F1 axis are the spectra of itraconazole, which illustrates a different measurement of diffusion for the drug than the polymer. This illustrates clearly that the drug is not tightly bound to the polymer by strong intermolecular interactions. If there is any hydrogen bonding, the drug/polymer association would have to be flexible enough to allow ITZ movement along PVAL to account for the different rates of diffusion. Again, these results appear to support the previously mentioned concept where the PVAL is functioning as a polymer 

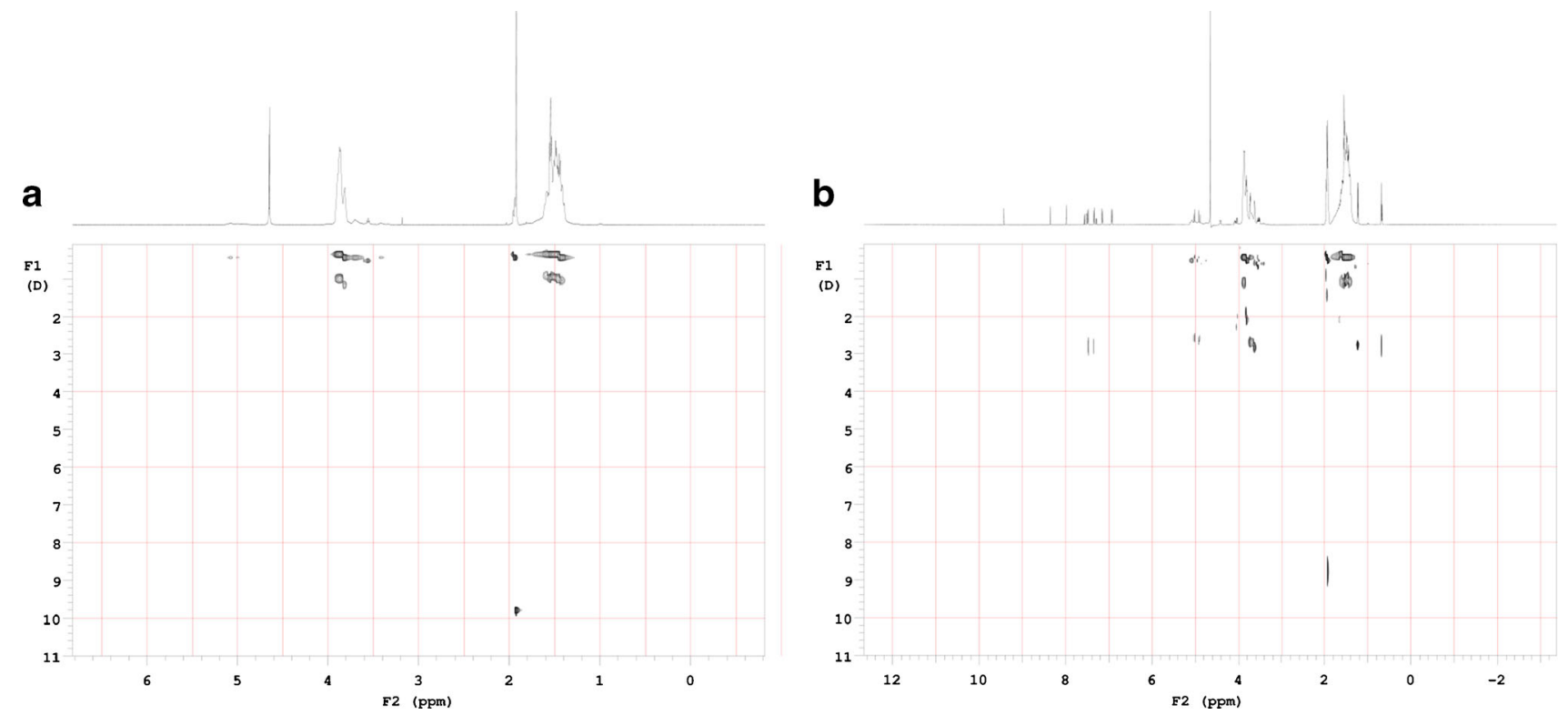

Fig. 6. DOSY maps of (a) PVAL 4-88 and (b) 20\% ITZ/80\% PVAL 4-88 amorphous dispersion

surfactant and creating some geometry in solution where the drug can associate to achieve supersaturation. Ideally, a third DOSY map of only ITZ in solution would be very useful to see if the interaction of the polymer changes the diffusion for the drug to illustrate a practical interaction between the two, but, unfortunately, the concentration of ITZ in solution was below the limit of detection for this analytical test. There is an area around 2 on the $\mathrm{F} 1$ axis that lies between the diffusion of PVAL 4-88 and ITZ that could possibly be a slower measured diffusion for ITZ that would indicate an interaction. However, it could also be a software interpretation error due to peaks of both PVAL 4-88 and ITZ both occurring in that area. Thus, the key conclusion that can be drawn from these results is that the concentration enhancing effect of PVAL on ITZ is likely the result of weak intermolecular interactions, such as van der
Waals forces/hydrophobic interactions, as it appears that strong intermolecular interactions between ITZ and PVAL do not occur in solution. This conclusion is supported by other research efforts that have looked at amorphous dispersions of ITZ with various polymeric carriers and concluded that hydrogen bonding was not the mechanism of action in solution between the drug and polymer $(18,20)$.

\section{Evaluation of Stability}

One of the main concerns to be addressed when creating amorphous dispersions is the stability of the matrix to prevent the drug from returning to its crystalline state within the delivery form before dosage (47-49). Physical stability of the

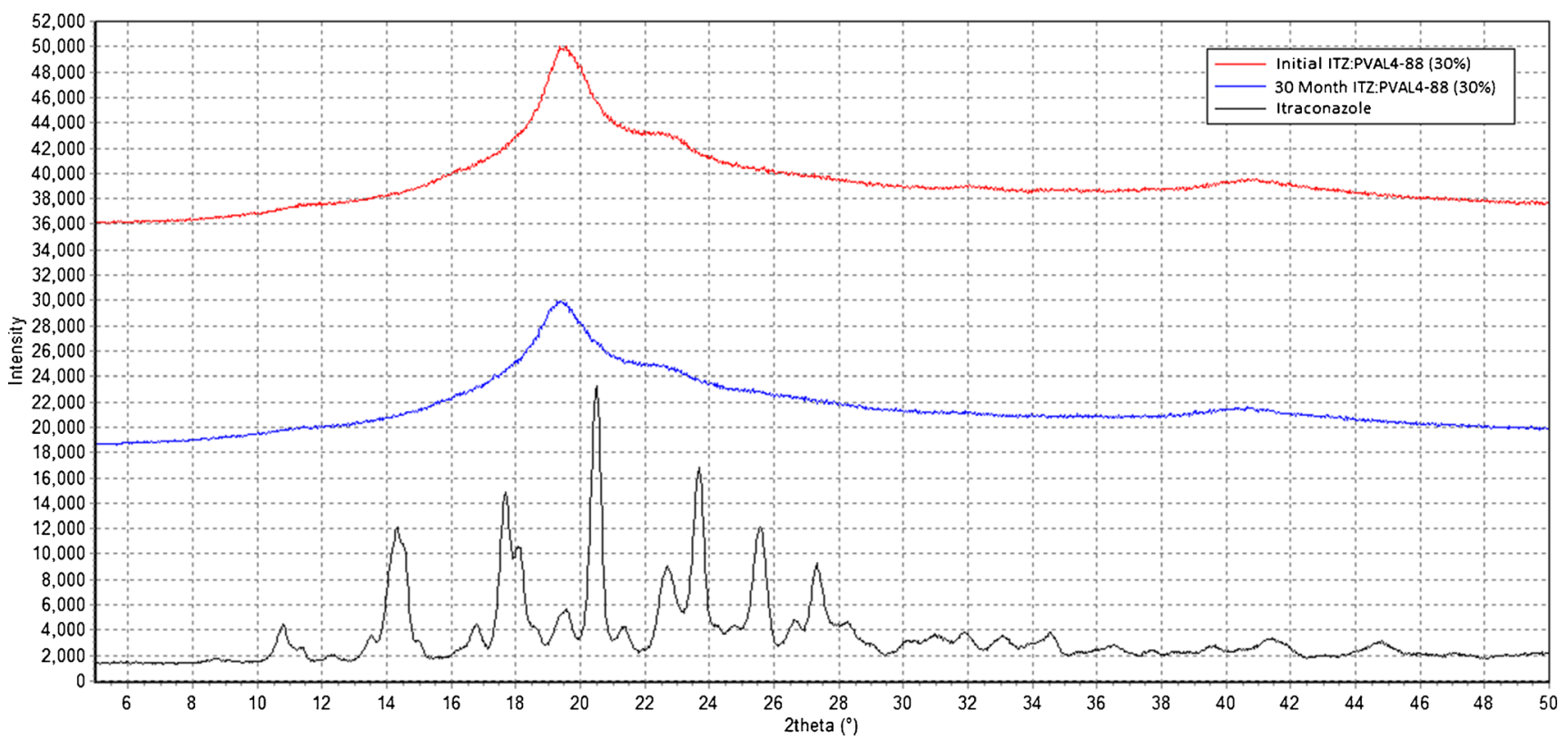

Fig. 7. XRD profiles of ITZ, initial and 30-month-old amorphous dispersions 


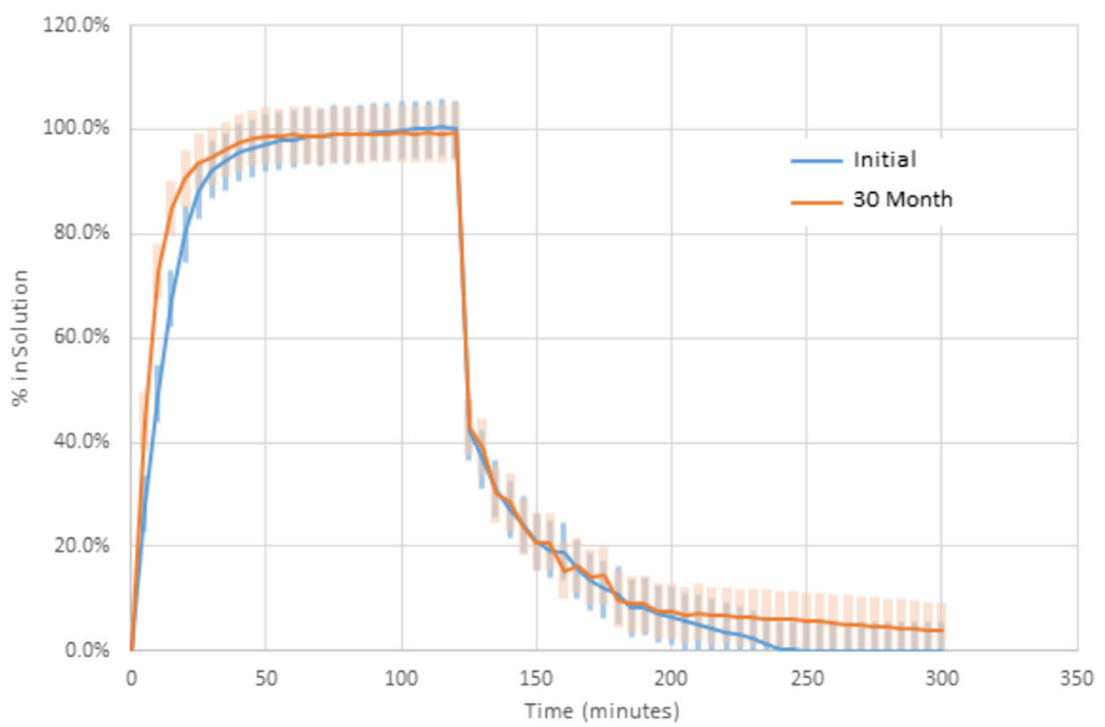

Fig. 8. pH change dissolution comparing initial and 30-month ambient storage amorphous dispersions of ITZ:PVAL 4-88 (30\% drug loading)

drug substance can be achieved through kinetic stabilization or freezing the amorphous drug within the polymeric carrier. As a good rule of thumb, this can be achieved by having a $\mathrm{T}_{\mathrm{g}}$ of the composite matrix $50^{\circ} \mathrm{C}$ above ambient storage conditions (50). It can also be achieved by thermodynamic stability which is attained by molecular interactions that hamper mobility (51). Many amorphous dispersions are formulated in a metastable region where the mode of stabilization is a combination of both kinetic and thermodynamic mechanisms (47). Drug loading also influences stability because as loading is increased, at some point miscibility is exceeded and then drug molecule proximity within the polymer matrix becomes critical. Stability tends to decrease because recrystallization becomes more probable as drug molecules are increasingly found in close proximity to other drug molecules.

The drug loading portion of the current study concluded that $30 \%$ ITZ in PVAL 4-88 is probably closer to the optimal drug loading than the other drug loadings tested. A container (without desiccant) containing milled amorphous intermediate powder of $30 \%$ ITZ was stored at ambient conditions for 30 months. A new sample that was identical in formulation was prepared and compared to the 30 -month-old material by XRD and PLM. The XRD diffractogram is shown in Fig. 7.

Both the initial and 30 month XRD profiles contain no peaks that correspond with ITZ indicating that both are substantially amorphous. The major broad peak in the amorphous dispersions at approximately 19 2-theta and minor peaks at approximately 23 and 41 2-theta are related to the crystallinity of the PVAL 4-88. No visual crystal structures were observed in either sample by PLM. Since it is possible to have crystals below the limit of detection of XRD and PLM, a dissolution study was conducted to confirm the dissolution performance of the amorphous compositions. These results are presented in Fig. 8. As stated in the methods section, the first $120 \mathrm{~min}$ of the dissolution was performed in 0.1 N HCL after which a buffer is added to change to a neutral $\mathrm{pH}$. Thus, in the acidic environment full release of ITZ is realized after which the $\mathrm{pH}$ change resulted in precipitation of ITZ. Since only two dissolution profiles are compared in this figure, error bars were included to show the significance of any variation.

The dissolution performance in $0.1 \mathrm{~N} \mathrm{HCl}$ of the two compositions is comparable with the 30 -month-old composition performing slightly better. Two different lots of PVAL 488 were used to make these compositions, which could explain the slight variance. Another possibility is that the stored powder could have had some minor water absorption that would allow for better wetting and faster release. Since the purpose of the test was to determine crystal growth during storage, the reason for this variance was not investigated. After the $\mathrm{pH}$ change, the two compositions performed similarly with the two curves staying within the error bars. Because the dissolution performance is similar, it can be concluded that the solidstate properties of ITZ in the PVAL matrix did not change during storage. Therefore, dissolution performance and XRD results demonstrate acceptable real-time ambient stability for the lead ITZ:PVAL 4-88 composition.

\section{CONCLUSION}

PVAL has been further investigated as a solubility enhancing polymer in amorphous solid dispersions as enabled by KinetiSol ${ }^{\circledR}$ Dispersing. PVAL 4-88 was confirmed to provide the best solubility enhancement and in solution stability as compared with $5-88,8-88$ and $18-88$ presenting a trend that lower molecular weights function better than higher molecular weight polyvinyl alcohols. In a drug loading study, 20, 30, and $40 \%$ ITZ drug loads achieve full drug release in $0.1 \mathrm{~N} \mathrm{HCl}$ in $2 \mathrm{~h}$, and were able to retard the return to the crystalline equilibrium solubility after the $\mathrm{pH}$ change in the expected rank order of $10 \%>20 \%>30 \%>40 \%>50 \%$. The $30 \%$ loading was selected as optimal. FTIR indicated some hydrogen bonding could occur in the solid state, but likely other molecular forces play a larger part in storage stability. DOSY indicated that weak molecular interactions are the likely cause of solution stability of amorphous ITZ. A 30 month stability study showed the optimal ITZ:PVAL 4-88 amorphous 
dispersion remained entirely amorphous as determined by XRD and PLM and the dissolution performance was unchanged.

\section{ACKNOWLEDGMENTS}

The authors wish to gratefully acknowledge the financial support of Merck Millipore.

\section{REFERENCES}

1. Dahan A, Miller JM, Amidon GL. Prediction of solubility and permeability class membership: provisional BCS classification of the world's top oral drugs. AAPS J. 2009;11(4):740-6.

2. Takagi $\mathrm{T}$ et al. A provisional biopharmaceutical classification of the top 200 oral drug products in the United States, Great Britain, Spain, and Japan. Mol Pharm. 2006;3(6):631-43.

3. Ku MS, Dulin W. A biopharmaceutical classification-based Right-First-Time formulation approach to reduce human pharmacokinetic variability and project cycle time from First-InHuman to clinical Proof-Of-Concept. Pharm Dev Technol. 2012;17(3):285-302.

4. Vasconcelos T, Sarmento B, Costa P. Solid dispersions as strategy to improve oral bioavailability of poor water soluble drugs. Drug Discov Today. 2007;12(23-24):1068-75.

5. Brough $\mathrm{C}$, Williams Iii RO. Amorphous solid dispersions and nano-crystal technologies for poorly water-soluble drug delivery. Int J Pharm. 2013;453(1):157-66.

6. Vo CL-N, Park C, Lee B-J. Current trends and future perspectives of solid dispersions containing poorly water-soluble drugs. Eur J Pharm Biopharm. 2013;85(3):799-813.

7. Van den Mooter G. The use of amorphous solid dispersions: a formulation strategy to overcome poor solubility and dissolution rate. Drug Discov Today Technol. 2012;9(2):e79-85.

8. Six K et al. Clinical study of solid dispersions of itraconazole prepared by hot-stage extrusion. Eur J Pharm Sci. 2005;24(2):179-86.

9. Miller DA et al. Enhanced in vivo absorption of itraconazole via stabilization of supersaturation following acidic-to-neutral $\mathrm{pH}$ transition. Drug Dev Ind Pharm. 2008;34(8):890-902.

10. Mellaerts $\mathrm{R}$ et al. Increasing the oral bioavailability of the poorly water soluble drug itraconazole with ordered mesoporous silica. Eur J Pharm Biopharm. 2008;69(1):223-30.

11. Verreck $\mathrm{G}$ et al. Characterization of solid dispersions of itraconazole and hydroxypropylmethylcellulose prepared by melt extrusion-part I. Int J Pharm. 2003;251(1-2):165-74.

12. Six $\mathrm{K}$ et al. Characterization of solid dispersions of itraconazole and hydroxypropylmethylcellulose prepared by melt extrusion, part II. Pharm Res. 2003;20(7):1047-54.

13. Verreck $\mathrm{G}$ et al. The effect of pressurized carbon dioxide as a temporary plasticizer and foaming agent on the hot stage extrusion process and extrudate properties of solid dispersions of itraconazole with PVP-VA 64. Eur J Pharm Sci. 2005;26(3):349-58.

14. Janssens $\mathrm{S}$ et al. The use of a new hydrophilic polymer, Kollicoat $\mathrm{IR}^{\circledR}$ in the formulation of solid dispersions of Itraconazole. Eur $\mathrm{J}$ Pharm Sci. 2007;30(3):288-94.

15. Miller DA et al. Targeted intestinal delivery of supersaturated itraconazole for improved oral absorption. Pharm Res. 2008:25(6):1450-9.

16. DiNunzio JC et al. Production of advanced solid dispersions for enhanced bioavailability of itraconazole using KinetiSol ${ }^{\circledR}$ Dispersing. Drug Dev Ind Pharm. 2010;36(9):1064-78.

17. Zhang $\mathrm{K}$ et al. Increased dissolution and oral absorption of itraconazole/Soluplus extrudate compared with itraconazole nanosuspension. Eur J Pharm Biopharm. 2013;85(3):1285-92.

18. DiNunzio JC et al. Amorphous compositions using concentration enhancing polymers for improved bioavailability of itraconazole. Mol Pharm. 2008;5(6):968-80.

19. Miller DA, et al. KinetiSol: a new processing paradigm for amorphous solid dispersion systems. Drug Dev Deliv. 2012;12(9).
20. Hughey JR et al. Preparation and characterization of fusion processed solid dispersions containing a viscous thermally labile polymeric carrier. Int J Pharm. 2012;438(1):11-9.

21. Law D et al. Physicochemical considerations in the preparation of amorphous ritonavir-poly(ethylene glycol) 8000 solid dispersions. J Pharm Sci. 2001;90(8):1015-25.

22. Bley H, Fussnegger B, Bodmeier R. Characterization and stability of solid dispersions based on PEG/polymer blends. Int J Pharm. 2010;390(2):165-73.

23. Newa $\mathrm{M}$ et al. Preparation, characterization and in vivo evaluation of ibuprofen binary solid dispersions with poloxamer 188 . Int J Pharm. 2007;343(1):228-37.

24. Surikutchi BT et al. Drug-excipient behavior in polymeric amorphous solid dispersions. J Excipients Food Chem. 2013;4(3):7094.

25. Zhu Q, Taylor LS, Harris MT. Evaluation of the microstructure of semicrystalline solid dispersions. Mol Pharm. 2010;7(4):1291300.

26. Galindo-Rodriguez S et al. Physicochemical parameters associated with nanoparticle formation in the salting-out, emulsificationdiffusion, and nanoprecipitation methods. Pharm Res. 2004;21(8):1428-39.

27. Sahoo SK et al. Residual polyvinyl alcohol associated with poly (D, L-lactide-co-glycolide) nanoparticles affects their physical properties and cellular uptake. J Control Release. 2002;82(1):105-14.

28. Mu L, Feng S. A novel controlled release formulation for the anticancer drug paclitaxel $\left(\mathrm{Taxol}^{\circledR}\right)$ : PLGA nanoparticles containing vitamin E TPGS. J Control Release. 2003;86(1):33-48.

29. Alcohol M-P. Technical brochure. Sulzbach: Clariant GmgH; 1999. http://docslide.us/documents/mowiol.html.

30. Torchilin VP. Structure and design of polymeric surfactant-based drug delivery systems. J Control Release. 2001;73(2):137-72.

31. Young TJ et al. Rapid expansion from supercritical to aqueous solution to produce submicron suspensions of water-insoluble drugs. Biotechnol Prog. 2000;16(3):402-7.

32. Sun DD, Lee PI. Probing the mechanisms of drug release from amorphous solid dispersions in medium-soluble and mediuminsoluble carriers. J Control Release. 2015;211:85-93.

33. Rambali B et al. Itraconazole formulation studies of the meltextrusion process with mixture design. Drug Dev Ind Pharm. 2003;29(6):641-52.

34. Craig DQ. The mechanisms of drug release from solid dispersions in water-soluble polymers. Int J Pharm. 2002;231(2):131-44.

35. Mansur HS et al. FTIR spectroscopy characterization of poly (vinyl alcohol) hydrogel with different hydrolysis degree and chemically crosslinked with glutaraldehyde. Mater Sci Eng C. 2008;28(4):539-48.

36. Xue B, Zhang J, Zhou T. Moving-window two-dimensional correlation infrared spectroscopic study on the dissolution process of poly (vinyl alcohol). Anal Bioanal Chem. 2015;407(29):8765-71.

37. Michaels AS, Bixler HJ. Solubility of gases in polyethylene. J Polym Sci. 1961;50(154):393-412.

38. Kazarian S, Martirosyan G. Spectroscopy of polymer/drug formulations processed with supercritical fluids: in situ ATR-IR and Raman study of impregnation of ibuprofen into PVP. Int J Pharm. 2002;232(1):81-90.

39. van der Weerd J, Kazarian SG. Combined approach of FTIR imaging and conventional dissolution tests applied to drug release. J Control Release. 2004;98(2):295-305.

40. Konno H, Taylor LS. Influence of different polymers on the crystallization tendency of molecularly dispersed amorphous felodipine. J Pharm Sci. 2006;95(12):2692-705.

41. Nesseem DI. Formulation and evaluation of itraconazole via liquid crystal for topical delivery system. J Pharm Biomed Anal. 2001;26(3):387-99.

42. Garg A, Sachdeva R, Kapoor G. Comparison of crystalline and amorphous carriers to improve the dissolution profile of water insoluble drug itraconazole. Int J Pharm Bio Sci. 2013;4(1):934. 2013. 948.

43. Calderini A, Pessine F. Synthesis and characterization of inclusion complex of the vasodilator drug minoxidil with $\beta$-cyclodextrin. J Incl Phenom Macrocycl Chem. 2008;60(3-4):369-77.

44. Crescenzi V et al. Synthesis and partial characterization of hydrogels obtained via glutaraldehyde crosslinking of acetylated 
chitosan and of hyaluronan derivatives. Biomacromolecules. 2003;4(4):1045-54.

45. Trefi $\mathrm{S}$ et al. The usefulness of 2D DOSY and 3D DOSY-COSY 1H NMR for mixture analysis: application to genuine and fake formulations of sildenafil (Viagra). Magn Reson Chem. 2009;47(S1):S163-73.

46. Laurienzo $\mathrm{P}$ et al. Novel alginate-acrylic polymers as a platform for drug delivery. J Biomed Mater Res A. 2006;78(3):523-31.

47. Qian F, Huang J, Hussain MA. Drug-polymer solubility and miscibility: Stability consideration and practical challenges in amorphous solid dispersion development. J Pharm Sci. 2010;99(7):2941-7.
48. Huang J, Wigent RJ, Schwartz JB. Drug-polymer interaction and its significance on the physical stability of nifedipine amorphous dispersion in microparticles of an ammonio methacrylate copolymer and ethylcellulose binary blend. J Pharm Sci. 2008;97(1):25162.

49. Ambike AA, Mahadik K, Paradkar A. Stability study of amorphous valdecoxib. Int J Pharm. 2004;282(1):151-62.

50. Hancock BC, Shamblin SL, Zografi G. Molecular mobility of amorphous pharmaceutical solids below their glass transition temperatures. Pharm Res. 1995;12(6):799-806.

51. Chokshi RJ et al. Stabilization of low glass transition temperature indomethacin formulations: impact of polymer-type and its concentration. J Pharm Sci. 2008;97(6):2286-98. 